\title{
Neutrophil Lymphocyte Ratio and Cardiovascular Disease Risk: A Systematic Review and Meta-Analysis
}

\author{
Teeranan Angkananard, ${ }^{1,2}$ Thunyarat Anothaisintawee $\left(\mathbb{D},{ }^{3}\right.$ Mark McEvoy, \\ John Attia $(1),{ }^{4}$ and Ammarin Thakkinstian $\left(\mathbb{1}^{1}\right.$ \\ ${ }^{1}$ Section for Clinical Epidemiology and Biostatistics, Faculty of Medicine, Ramathibodi Hospital, \\ Mahidol University, Bangkok, Thailand \\ ${ }^{2}$ Division of Cardiovascular Medicine, Department of Medicine, Faculty of Medicine, \\ HRH Princess Maha Chakri Sirindhorn Medical Center, Srinakharinwirot University, Nakhon Nayok, Thailand \\ ${ }^{3}$ Department of Family Medicine, Faculty of Medicine, Ramathibodi Hospital, Mahidol University, Bangkok, Thailand \\ ${ }^{4}$ Center for Clinical Epidemiology and Biostatistics, The School of Medicine and Public Health, \\ The University of Newcastle, Newcastle, NSW, Australia
}

Correspondence should be addressed to Thunyarat Anothaisintawee; thunyarat.ano@mahidol.ac.th

Received 28 June 2018; Revised 13 September 2018; Accepted 28 October 2018; Published 11 November 2018

Academic Editor: Pablo Avanzas

Copyright (C) 2018 Teeranan Angkananard et al. This is an open access article distributed under the Creative Commons Attribution License, which permits unrestricted use, distribution, and reproduction in any medium, provided the original work is properly cited.

Objective. This systematic review aimed to measure the association between neutrophil lymphocyte ratio (NLR) and cardiovascular disease (CVD) risk. Methods. Relevant studies were identified from Medline and Scopus databases. Observational studies with NLR as a study factor were eligible for review. The outcomes of interest were any type of CVD including acute coronary syndrome, coronary artery disease, stroke, or a composite of these cardiovascular events. Mean differences in NLR between CVD and nonCVD patients were pooled using unstandardized mean difference (USMD). Odds ratios of CVD between high and low NLR groups were pooled using a random effects model. Results. Thirty-eight studies $(\mathrm{n}=76,002)$ were included. High NLR was significantly associated with the risks of CAD, ACS, stroke, and composite cardiovascular events with pooled ORs of 1.62 (95\% CI: 1.38-1.91), 1.64 (95\% CI: 1.30, 2.05), 2.36 (95\% CI: 1.44, 2.89), and 3.86 (95\% CI: 1.73, 8.64), respectively. In addition, mean NLRs in CAD, ACS, and stroke patients were significantly higher than in control groups. Conclusion. High NLR was associated with CAD, ACS, stroke, and composite cardiovascular events. Therefore, NLR may be a useful CVD biomarker.

\section{Introduction}

Cardiovascular diseases (CVD) are the leading causes of mortality worldwide $[1,2]$. Approximately 17.7 million people died from CVD in 2015, with one-third due to coronary heart disease (CHD) and stroke. In addition, CVD carries a high economic burden, costing about \$316.1 billion/year [3]. Therefore, risk stratification and prognostication in CVD are important so that individuals at high risks can be accurately targeted for prevention.

The inflammatory response is a key mechanism in the pathogenesis of atherosclerosis and its progression [4]. Neutrophils secrete inflammatory mediators that can cause vascular wall degeneration. Conversely, lymphocytes regulate the inflammatory response and thus have an antiatherosclerotic role. Therefore, the neutrophil to lymphocyte ratio (NLR) has been proposed as an inflammatory biomarker [5] and potential predictor of risk and prognosis in CVD.

A previous systematic review suggested prognostic impacts of NLR on all-cause mortality and recurrent cardiovascular events (CVEs) among CHD patients [68]. However, the effect of NLR on CVD is still unclear and thus far, there has been no meta-analysis quantifying these associations. We therefore conducted a systematic review and meta-analysis of observational studies aiming to explore and quantify the association between NLR and CVD risk. 


\section{Methods}

A systematic review of observational studies was performed according to the MOOSE guidelines [9]. This review has been registered in PROSPERO (no. CRD42016043554).

2.1. Search Strategy. Relevant studies were identified from Medline and Scopus databases since their inceptions to 19th August 2018. The following search terms were used: 'cardiovascular disease', 'coronary heart disease', 'coronary artery disease', 'myocardial infarction', 'angina', 'stroke', 'cerebrovascular', and 'neutrophil lymphocyte ratio'. The search strategies for both databases are presented in Supplementary Appendix. Reference lists of included studies and previous systematic reviews were also explored to identify eligible studies not located using the database searches.

2.2. Selection of Studies. Identified studies were independently selected based on titles and abstracts by two reviewers (T.A.1 and T.A.2). Full articles were retrieved if a decision could not be made from the titles and abstracts. Disagreements were resolved by consensus and discussion with a third party (A.T.). Inclusion criteria were as follows: (1) any type of observational study or baseline randomized controlled trial published in English; (2) including adult patients aged $\geq 18$ years; (3) having NLR as a study factor; (4) having CVD as outcome of interest; (5) providing sufficient data for pooling, i.e., number of patients, mean and standard deviation of NLR between CVD and non-CVD patients, and/or numbers of contingency cells between low/high NLR and CVD/nonCVD. For studies with insufficient data, up to 3 attempts to contact corresponding authors were made.

2.3. Outcome Measurement. The outcomes of interest were coronary artery disease including chronic stable angina, acute coronary syndrome, cerebrovascular diseases including ischemic/hemorrhagic stroke or transient ischemic attack, and cardiovascular (CV) death, as well as a composite CVD outcome of these.

2.4. Study Factor. The study factor was NLR, measured from the complete blood count according to the original studies, and was calculated by dividing neutrophil count by lymphocyte count.

2.5. Data Extraction. All data were independently extracted by 2 reviewers (T.A.1 and T.A.2). Discrepancies between two reviewers were resolved through discussion with the senior author (A.T.). Characteristics of the included studies [i.e., study design, setting, mean age, body mass index (BMI), and mean NLR of study participants, percentages having diabetes mellitus, hypertension (HT), dyslipidemia (DLP), and smoking] and cut-off values of NLR for studies assessing the effect of high versus low NLR were extracted. Incidence or prevalence of CVD and non-CVD patients between high and low NLR was extracted for pooling odds ratio (OR). For studies that did not provide these estimates, risk ratios [e.g., OR, relative risk (RR), and HR] along with their 95\% confidence intervals (CIs) were extracted instead. To complete data analysis of continuous variables data, the mean difference was calculated and used as one of the summary effect sizes; the number of patients and mean and standard deviation (SD) of NLR between CVD and nonCVD patients were extracted for pooling mean difference of NLR.

2.6. Risk of Bias Assessment. Risk of bias assessments of included studies were independently assessed by two reviewers (T.A.1 and T.A.) using the Newcastle-Ottawa quality assessment scale [49]. NOS has three domains to assess, which are (1) selection of study groups (4 items), (2) comparability of groups (2 items), and (3) ascertainment of exposure and outcome (3 items). Each item in the 3 domains was graded as 0 to 1 with a total score ranging from 0 to 9; higher total score reflected higher quality or lower risk of bias.

Since the NOS does not have criteria for judging crosssectional studies, criteria for cohort study were adapted to assess the risk of bias for cross-sectional study. Two items in the domain of ascertainment of outcome (i.e., adequate duration of follow-up and adequate follow-up of cohort) were excluded because they are not relevant for cross-sectional studies. Therefore, the total score for this design ranged from 0 to 7 , instead of 0 to 9 .

2.7. Statistical Analysis. For comparison of mean NLR between CVD and non-CVD groups, mean differences in NLR between CVD and non-CVD groups were estimated and were pooled using the unstandardized mean difference (USMD). For categorical outcomes, cut-off points of NLR for each study were recategorized into low versus high NLR groups as follows: For those studies with four NLR categories, two lower and two higher NLR categories were classified as low and high NLR groups, respectively; for three categories, the low and intermediate groups were combined and assigned as low NLR whereas the last group was assigned as high NLR. Odds ratio of having CVD between high and low NLR groups of each study was then estimated and pooled using a fixed effects model (inverse variance method) if there was no heterogeneity between studies; otherwise, a random-effects model (DerSimonian and Laird) was applied.

Cochrane's Q test and the degree of heterogeneity $\left(\mathrm{I}^{2}\right.$ statistic) were applied to assess heterogeneity between studies. Heterogeneity between studies was considered, if P-value from Cochrane's Q test was less than 0.10 or degree of heterogeneity was equal to or greater than $25 \%$. Sources of heterogeneity were explored by considering the covariables (i.e., mean age, BMI, and smoking history) one by one in a meta-regression model. Subgroup analysis was further performed according to the covariables which could decrease $\mathrm{Tau}^{2}$ greater than $50 \%$.

Publication bias was explored using Egger's test and funnel plot. If there was asymmetry of funnel plot, a contour enhanced funnel plot was applied to explore the cause of asymmetry. 


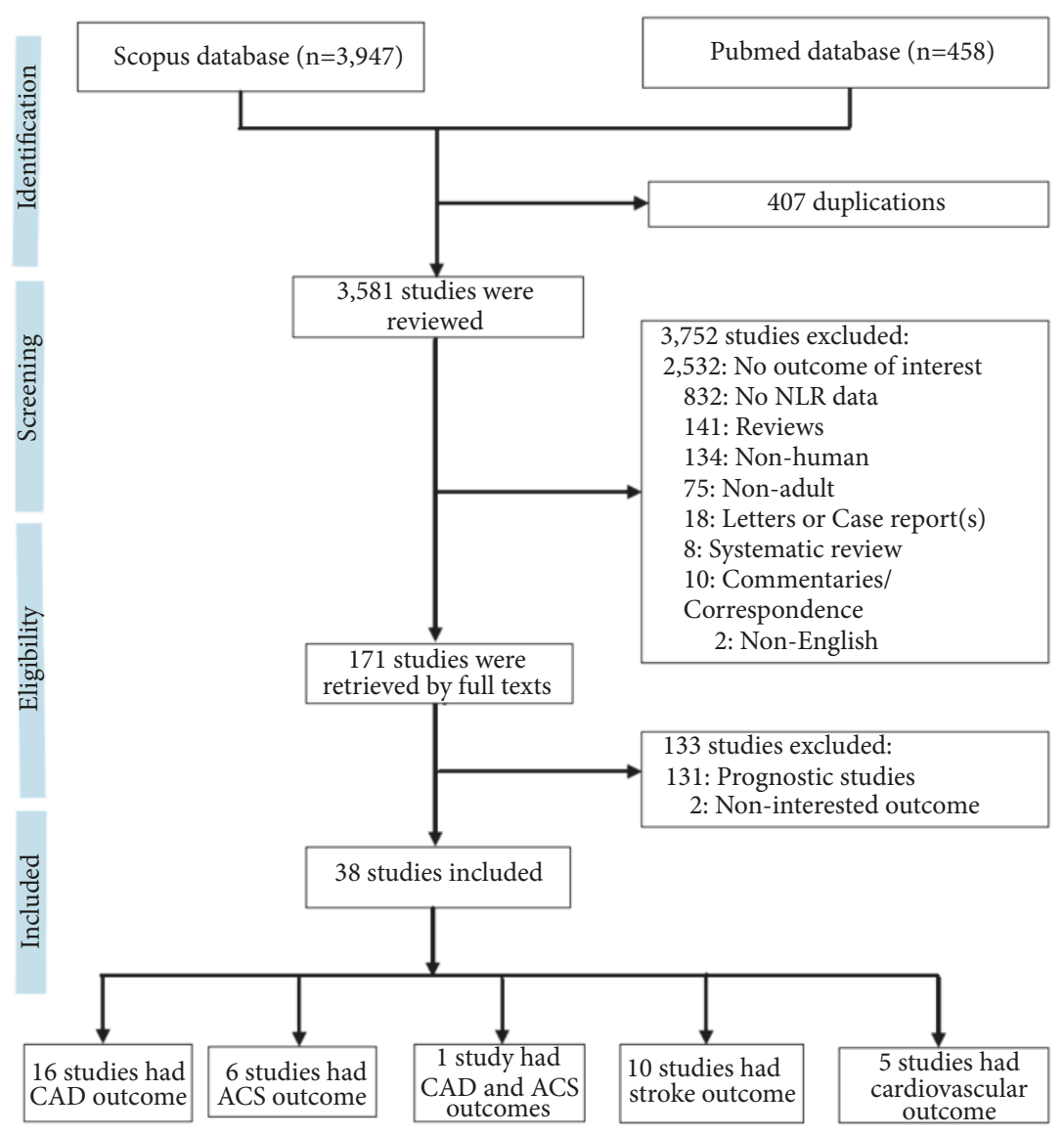

FIGURE 1: Flow chart of study selection.

All analyses were performed using STATA software, version 15.0 (StataCorp LP, College Station, TX, USA). A twosided test with $\mathrm{P}$-value $<0.05$ was considered for statistical significance except for the heterogeneity test, in which Pvalue $<0.1$ was applied.

\section{Results}

A total of 4,405 relevant studies were identified from Medline and Scopus databases; see Figure 1. Among them, 4,367 studies were excluded leaving 38 studies [10-47] eligible for review. These consisted of 76,002 participants from 11 case-control, 9 cohort, and 18 cross-sectional studies. There were $16,6,10$, and 5 studies that, respectively, reported stable CAD [10-17, 19-26], ACS [27-32], stroke [33-42], and composite CVD outcomes [43-47]. One study [18] reported both CAD and ACS.

Characteristics of included studies are illustrated in Table 1. Mean age of study participants ranged from 34.9 to 73.2 years. Some studies focused on patients with specific diseases including diabetic patients in 6 studies $[14,16,23$, $30,43,44]$, gastric cancer in 1 study [40], CKD patients in 2 studies, and HIV patients in 1 study [47]. Percentages of patients having DM, HT, and DLP and smoking ranged within $0 \%-100 \%, 5.9 \%-80.9 \%, 12.4 \%-83.9 \%$, and $0 \%-45.8 \%$, respectively.
3.1. Risk of Bias Assessment. Results of the risk of bias assessments are presented in Supplementary Tables 1 and 2. The total scores ranged from 4 to 7,6 to 8 , and 3 to 7 for case-control, cohort, and cross-sectional studies, respectively. For case-control study, nearly all studies had high risk of bias for definition of case and all for nonresponse rate but had low risk of bias for representativeness of cases, definition of controls, assessment of exposure, and same method of outcome ascertainment for cases and controls. For cohort and cross-sectional studies, almost all studies had low risk of bias for ascertainment of exposure and assessment of outcome.

3.2. CAD Outcome. Among 17 studies of 8,988 subjects, 4 studies $[14,16,20,23]$ reported ORs of high versus low NLR, 8 studies [10-12, 17, 19, 21, 22, 26] reported mean differences of NLR between CAD and non-CAD patients, and 5 studies $[13,15,18,24,25]$ reported both.

3.2.1. High versus Low NLR. A total of 7,405 patients were included in pooling. Study design was cross-sectional in most studies (8/9), while one [18] study was prospective. Contingency data of NLR and CAD groups are presented in Table 2. The NLR cut-off points ranged from 1.80 to 2.60. Estimated ORs from those 9 studies were moderately varied $\left(\right.$ Chi-square $=17.01, \mathrm{P}$-value $=0.03, \mathrm{I}^{2}=53.0 \%$ with a 


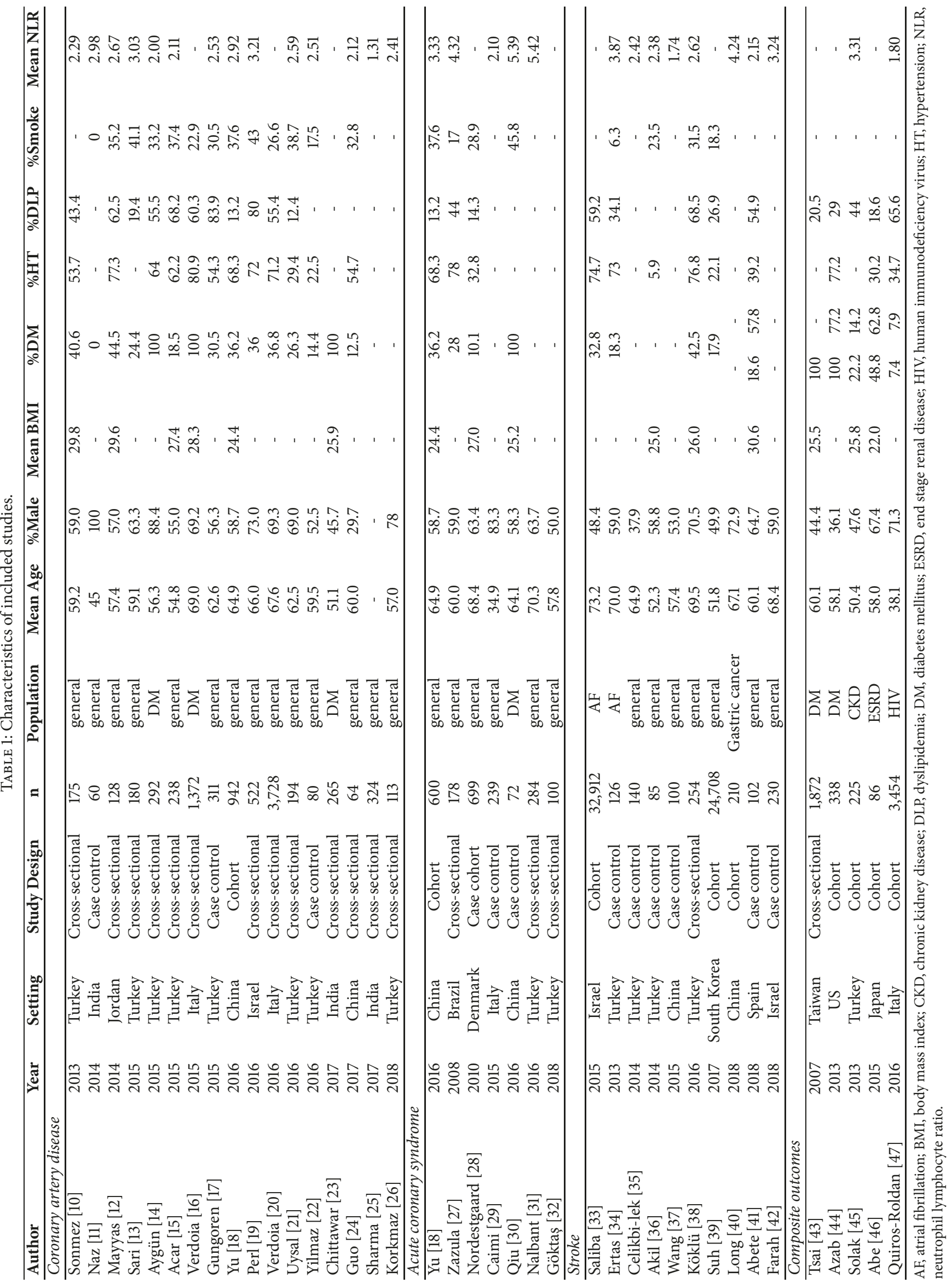


TABLE 2: Pooled odds ratio of cardiovascular between high and low NLR.

\begin{tabular}{|c|c|c|c|c|c|c|c|}
\hline \multirow{2}{*}{ Author } & \multirow{2}{*}{ Year } & \multirow{2}{*}{$\begin{array}{c}\text { NLR } \\
\text { Cutoff }\end{array}$} & \multicolumn{2}{|c|}{ CVD } & \multicolumn{2}{|c|}{ Non-CVD } & \multirow{2}{*}{ OR (95\%CI) } \\
\hline & & & Low NLR & High NLR & Low NLR & High NLR & \\
\hline \multicolumn{8}{|c|}{ Coronary artery disease } \\
\hline Sari [13] & 2015 & 2.30 & - & - & - & - & $1.51(1.15,2.00)$ \\
\hline Aygün [14] & 2015 & 2.05 & 40 & 56 & 109 & 87 & $1.75(1.07,2.88)$ \\
\hline Acar [15] & 2015 & 2.25 & - & - & - & - & $2.30(1.19,4.43)$ \\
\hline Verdoia [16] & 2015 & 2.03 & 349 & 783 & 103 & 137 & $1.69(1.27,2.24)$ \\
\hline Yu [18] & 2016 & 2.41 & - & - & - & - & $1.69(1.48,1.94)$ \\
\hline Verdoia [20] & 2016 & 1.80 & 682 & 2172 & 251 & 633 & $1.26(1.07,1.49)$ \\
\hline Chittawar [23] & 2017 & 2.60 & 2 & 8 & 197 & 58 & $13.59(2.81,65.76)$ \\
\hline Guo [24] & 2017 & 2.45 & - & - & - & - & $2.01(0.88,4.63)$ \\
\hline Sharma [25] & 2017 & 2.13 & - & - & - & - & $1.49(0.94,2.37)$ \\
\hline Pooled OR (95\% CI) & & & & & & & $1.62(1.38,1.91)$ \\
\hline \multicolumn{8}{|c|}{ Acute coronary syndrome } \\
\hline $\mathrm{Yu}[18]$ & 2016 & 2.42 & - & - & - & - & $1.65(1.43,1.90)$ \\
\hline Zazula [27] & 2008 & 5.70 & - & - & - & - & $4.51(1.51,13.45)$ \\
\hline Nordestgaard [28] & 2010 & - & - & - & - & - & $1.52(0.83,2.79)$ \\
\hline Caimi [29] & 2015 & 2.19 & 39 & 43 & 11 & 12 & $1.01(0.40,2.55)$ \\
\hline Göktaş [32] & 2018 & 3.0 & 40 & 23 & 26 & 11 & $1.36(0.57,3.25)$ \\
\hline Pooled OR (95\% CI) & & & & & & & $1.64(1.30-2.05)$ \\
\hline \multicolumn{8}{|l|}{ Stroke } \\
\hline Saliba [33] & 2015 & 3.15 & 649 & 332 & 24049 & 7882 & $1.56(1.36,1.79)$ \\
\hline Ertas [34] & 2013 & 3.17 & 20 & 19 & 64 & 23 & $2.64(1.20,5.81)$ \\
\hline Akil [36] & 2014 & - & - & - & - & - & $8.95(1.88,42.61)$ \\
\hline Suh [39] & 2017 & 3.00 & 23,530 & 219 & 936 & 23 & $2.64(1.71,4.08)$ \\
\hline Pooled OR (95\% CI) & & & & & & & $2.36(1.44,3.89)$ \\
\hline \multicolumn{8}{|l|}{ Composite outcomes } \\
\hline Tsai [43] & 2007 & - & - & - & - & - & $1.52(0.96,2.40)$ \\
\hline Azab [44] & 2013 & 2.40 & 20 & 206 & 23 & 89 & $2.66(1.39,5.09)$ \\
\hline Solak [45] & 2013 & 2.80 & 3 & 63 & 109 & 50 & $45.78(13.71,152.85)$ \\
\hline Abe [46] & 2015 & 3.67 & 10 & 26 & 33 & 17 & $5.05(1.98,12.86)$ \\
\hline Quiros-Roldan [47] & 2016 & 1.20 & 22 & 90 & 1091 & 2251 & $1.98(1.24,3.18)$ \\
\hline Pooled OR (95\% CI) & & & & & & & $3.86(1.73,8.64)$ \\
\hline
\end{tabular}

CI, confidence interval; CVD, cardiovascular disease; NLR, neutrophil lymphocyte ratio; OR, odds ratio.

pooled OR of 1.62 (95\% CI: 1.38-1.91)); see Figure 2(a) and Supplementary Figure 1A.

Sources of heterogeneity were also explored. Only race (Caucasian versus Asian) and age ( $\leq$ versus $>65$ years) variables could reduce the $\mathrm{I}^{2}$ from $53 \%$ to $47.31 \%$ and $39.74 \%$, respectively, in the meta-regression model. A subgroup analysis was performed and showed that pooled ORs were higher in Asians (1.90; 95\% CI: 1.26, 2.87) than Caucasians (1.51; 95\% CI: $1.27,1.79)$ and greater in patients $\leq 65$ years $(1.73 ; 95 \%$ CI: $1.44,2.07)$ than patients $>65$ years $(1.43 ; 95 \% \mathrm{CI}: 1.08,1.89)$; see Supplementary Figures 1B and 1C.

3.2.2. Mean Difference of NLR. Thirteen studies reported mean difference in NLR between CAD and non-CAD patients (see Table 3). Most study designs (9/13 studies) were cross-sectional; three were case-control [11, 17, 22] and one [18] was cohort. USMD was 0.87 (95\% CI: 0.52,
1.22). Heterogeneity test and $\mathrm{I}^{2}$ suggested high heterogeneity between studies (Chi-square $=611.32 ; \mathrm{P}$-value $<0.001 ; \mathrm{I}^{2}$ = 98.0\%); see Figure 2(b) and Supplementary Figure 2A. Possible sources of heterogeneity were explored in a metaregression, but none of them could decrease the degree of heterogeneity. There was no evidence of publication bias from Egger's test $($ coefficient $=0.31 ; \mathrm{P}$-value $=0.253)$, but the funnel plot showed asymmetry; see Supplementary Figure 2B. A contour-enhanced funnel plot was therefore constructed, which suggested that asymmetry was more likely due to heterogeneity between studies; see Supplementary Figure 2C.

3.3. ACS Outcome. Among 7 studies of ACS outcome, 2 studies $[28,32]$ reported OR of high versus low NLR, 2 studies $[30,31]$ reported mean differences between ACS patients and controls, and 3 studies $[18,27,29]$ reported both. 


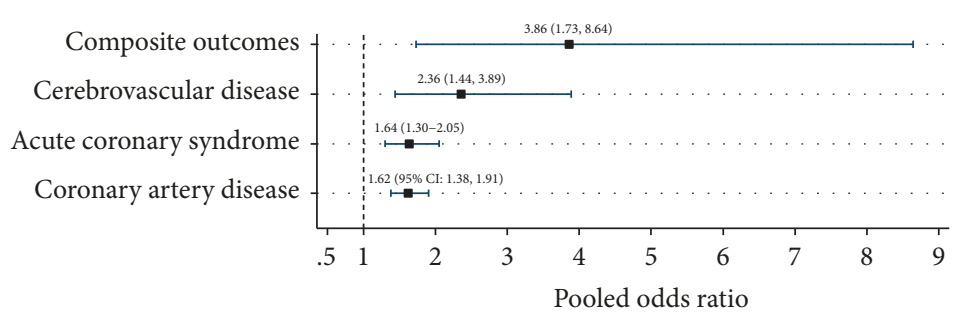

(a) High versus low NLR on CVD risk

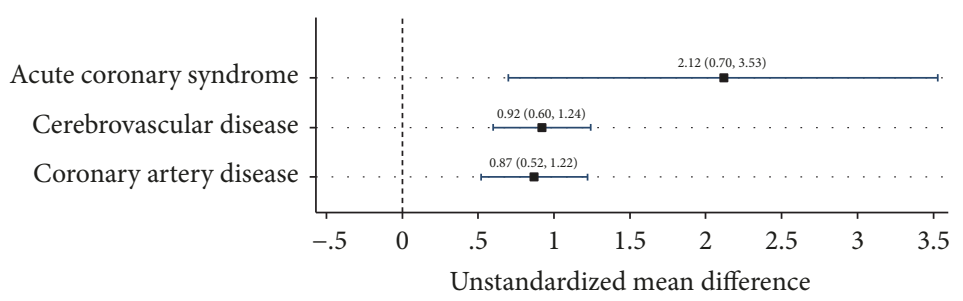

(b) Mean difference of NLR between CVD and non-CVD

FIGURE 2: Summary of pooled effect sizes of neutrophil lymphocyte ratio on cardiovascular risk.

3.3.1. High versus Low NLR. There were 1,816 subjects from 5 studies; see Table 2 . Study designs were cross-sectional $(n=2)$, case-control $(n=1)$, and cohort $(n=2)$. The cut-off points for defining high NLR ranged from 2.19 to 5.70. The pooled OR was 1.64 (95\% CI: 1.30, 2.05) with low heterogeneity (Chisquare $=4.59 ; \mathrm{P}$-value $=0.332 ; \mathrm{I} 2=12.8 \%$ ); see Figure $2(\mathrm{a})$ and Supplementary Figure 3A. There was no evidence of publication bias from Egger's test (coefficient $=0.49$; P-value $=0.943$ ), a funnel plot and contour-enhanced funnel plot; see Supplementary Figures 3B and 3C.

3.3.2. Mean Difference of NLR. Mean differences of NLR between ACS $(n=832)$ and non-ACS $(n=541)$ patients from five studies are presented in Table 3. Study designs were cross-sectional $(n=2)$, case-control $(n=2)$, and cohort $(n=1)$. The USMD of NLR was 2.12 (95\% CI: 0.70, 3.53) with high heterogeneity (Chi-square $=114.63 ;$ P-value $<0.001 ; \mathrm{I}^{2}=$ 96.5\%); see Figure 2(b) and Supplementary Figure 4A.

None of the covariables reduced the $\mathrm{I}^{2}$ after exploring sources of heterogeneity. There was no evidence of publication bias from Egger's test (coefficient $=4.19$; P-value $=$ 0.314 ), a funnel plot and contour-enhanced funnel plot; see Supplementary Figures 4B and 4C.

3.4. Stroke Outcome. Among 10 studies of 58,867 participants, 2 studies [33,39] reported OR of high versus low NLR, 6 studies [35, 37, 38, 40-42] reported mean differences between stroke patients and controls, and 2 studies [34, 36] reported both. Types of stroke were ischemic stroke and TIA.

3.4.1. High versus Low NLR. There were 24,769 stroke patients and 32,977 controls from 4 studies. Two studies [33, 34] included patients with atrial fibrillation (AF). Study designs were case-control $(n=2)$, and cohort $(n=2)$. Cut-off points of NLR ranged from 3.00 to 3.17. The pooled OR was 2.36 (95\% CI: 1.44, 3.89) with moderate heterogeneity (Chi-square
$=10.99, \mathrm{P}$-value $\left.=0.012, \mathrm{I}^{2}=72.7 \%\right)$; see Figure $2(\mathrm{a})$ and Supplementary Figure 5A.

By the meta-regression model, the population variable (AF versus non-AF) could reduce the $\mathrm{I}^{2}$ from $72.7 \%$ to $48.1 \%$. A subgroup analysis was evaluated and showed that pooled ORs were lower in AF (1.75; 95\% CI: 1.14, 2.68) than nonAF (3.83; 95\% CI: 1.27, 11.49); see Supplementary Figure 5B. The degrees of heterogeneities $\left(\mathrm{I}^{2}\right)$ were reduced to $40.0 \%$ and $54.2 \%$ in studies with the AF and non-AF, respectively. There was evidence of publication bias suggested from Egger's test (coefficient $=2.34 ;$ P-value $=0.045$ ) and funnel plot; however a contour-enhanced funnel plot suggested heterogeneity rather than publication bias; see Supplementary Figure 5C and $5 \mathrm{D}$.

3.4.2. Mean Difference of NLR. The USMD in NLR between stroke patients $(n=633)$ and controls $(n=614)$ from 8 studies was 0.92 (95\% CI: $0.60,1.24)$ with high heterogeneity (Chisquare $=121.86 ;$ P-value $\left.<0.001 ; \mathrm{I}^{2}=94.3 \%\right)$; see Table 3, Figure 2B, and Supplementary Figure 6A. The source of heterogeneity could not be identified from a meta-regression. There was no evidence of publication bias from Egger's test (coefficient $=0.82$; P-value $=0.931$ ), but a funnel plot showed asymmetry; see Supplementary Figure 6B. The contour enhanced funnel plot showed that most studies fell in the significant area and only one study fell in the nonsignificant area, so asymmetry might be due to publication bias; see Supplementary Figure 6C.

\subsection{Composite Outcomes}

3.5.1. High versus Low NLR. Five studies [43-47] were pooled for composite CVEs. Only one study [43] was cross-sectional and the remaining four studies were cohort. Different study endpoints were reported including ACS, stroke, and peripheral artery disease $(n=3)$ [45-47], ischemic stroke and CAD $(n=1)$ [43], and AMI and revascularization $(n=1)$ [44]. Cut-off 
TABLE 3: Mean difference of neutrophil lymphocyte ratio between CVD and non-CVD patients.

\begin{tabular}{|c|c|c|c|c|c|c|}
\hline \multirow{2}{*}{ Author } & \multirow{2}{*}{ Year } & \multicolumn{2}{|c|}{ CVD } & \multicolumn{2}{|c|}{ Non-CVD } & \multirow{2}{*}{ Mean differences $(95 \% \mathrm{CI})$} \\
\hline & & $\mathrm{N}$ & mean $[48]$ & $\mathrm{N}$ & mean $[48]$ & \\
\hline \multicolumn{7}{|c|}{ Coronary artery disease } \\
\hline Sonmez [10] & 2013 & 106 & $2.37(0.89)$ & 69 & $2.03(1.56)$ & $0.34(-0.07,0.75)$ \\
\hline $\mathrm{Naz}[11]$ & 2014 & 40 & $3.67(1.62)$ & 20 & $1.61(0.84)$ & $2.06(1.44,2.68)$ \\
\hline Mayyas [12] & 2014 & 60 & $2.61(0.17)$ & 68 & $2.72(0.19)$ & $-0.11(-0.17,-0.05)$ \\
\hline Sari [13] & 2015 & 100 & $3.70(2.60)$ & 80 & $2.2(1.7)$ & $1.50(0.87,2.13)$ \\
\hline Acar [15] & 2015 & 71 & $2.50(0.70)$ & 90 & $1.90(0.70)$ & $0.60(0.38,0.82)$ \\
\hline Gungoren [17] & 2015 & 261 & $2.73(1.07)$ & 50 & $1.51(0.42)$ & $1.22(1.05,1.39)$ \\
\hline Yu [18] & 2016 & 691 & $3.62(2.70)$ & 251 & $2.14(1.97)$ & $1.48(1.16,1.79)$ \\
\hline Perl [19] & 2016 & 170 & $3.44(2.90)$ & 352 & $3.00(2.50)$ & $0.44(-0.16,1.04)$ \\
\hline Uysal [21] & 2016 & 152 & $2.77(0.23)$ & 42 & $1.97(0.15)$ & $0.80(0.74,0.86)$ \\
\hline Yilmaz [22] & 2016 & 40 & $2.51(0.65)$ & 40 & $1.73(0.71)$ & $0.78(0.48,1.08)$ \\
\hline Guo [24] & 2017 & 31 & $2.93(1.82)$ & 33 & $2.11(0.79)$ & $0.82(0.13,1.52)$ \\
\hline Sharma [25] & 2017 & 225 & $5.60(4.50)$ & 99 & $4.30(3.80)$ & $1.30(0.35,2.25)$ \\
\hline Korkmaz [26] & 2018 & 63 & $2.66(0.86)$ & 50 & $2.10(0.53)$ & $0.56(0.30,0.82)$ \\
\hline USMD $(95 \% \mathrm{CI})$ & & & & & & $0.87(0.52,1.22)$ \\
\hline \multicolumn{7}{|c|}{ Acute coronary syndrome } \\
\hline Yu [18] & 2016 & 349 & $4.93(3.15)$ & 251 & $2.14(1.97)$ & $2.79(2.38,3.20)$ \\
\hline Nordestgaard [28] & 2008 & 133 & $4.77(3.83)$ & 45 & $3.00(1.60)$ & $1.77(0.97,2.57)$ \\
\hline Caimi [29] & 2015 & 123 & $2.38(0.87)$ & 116 & $1.82(0.71)$ & $0.56(0.36,0.76)$ \\
\hline Qiu [30] & 2016 & 38 & $8.10(6.44)$ & 34 & $2.37(1.19)$ & $5.73(3.64,7.82)$ \\
\hline Nalbant [31] & 2016 & 189 & $5.58(6.60)$ & 95 & $5.10(7.60)$ & $0.48(-1.32,2.28)$ \\
\hline USMD $(95 \% \mathrm{CI})$ & & & & & & $2.12(0.70,3.53)$ \\
\hline \multicolumn{7}{|l|}{ Stroke } \\
\hline Ertas [34] & 2013 & 39 & $5.60(3.40)$ & 87 & $3.10(2.10)$ & $2.50(1.35,3.66)$ \\
\hline Celikbilek [35] & 2014 & 70 & $2.97(0.53)$ & 70 & $1.88(0.40)$ & $1.09(0.96,1.23)$ \\
\hline Akil [36] & 2014 & 38 & $3.10(2.00)$ & 47 & $1.80(0.40)$ & $1.30(0.65,1.95)$ \\
\hline Wang [37] & 2015 & 50 & $1.40(0.83)$ & 50 & $1.40(0.41)$ & $0.00(-0.26,0.26)$ \\
\hline Köklü [38] & 2016 & 115 & $3.09(0.23)$ & 139 & $2.23(0.15)$ & $0.86(0.81,0.91)$ \\
\hline Long [40] & 2018 & 70 & $5.51(8.02)$ & 140 & $3.60(1.89)$ & $1.91(0.01,3.82)$ \\
\hline Abete [41] & 2018 & 51 & $2.30(0.50)$ & 51 & $2.00(0.30)$ & $0.30(0.14,0.46)$ \\
\hline Farah [42] & 2018 & 200 & $3.44(2.56)$ & 30 & $1.89(0.61)$ & $1.55(1.13,1.97)$ \\
\hline USMD (95\% CI) & & & & & & $0.92(0.60,1.24)$ \\
\hline
\end{tabular}

CI, confidence interval; CVD, cardiovascular disease; USMD, unstandardized mean difference.

points used for defining NLR ranged from 1.20 to 3.67. The pooled ORs across studies were 3.86 (95\% CI: 1.73, 8.64) with high heterogeneity (Chi-square $=29.97 ; \mathrm{P}$-value $=0.001 ; \mathrm{I}^{2}=$ $86.7 \%$ ); see Table 2, Figure 2(a), and Supplementary Figure 7A. Sources of heterogeneity were explored but none could reduce the $\mathrm{I}^{2}$. However, sensitivity analysis was performed according to the length of follow-up. Follow-up time ranged from 36 to 39 months in 3 cohort studies [45-47], while one study had longer follow-up time (48 months) [44]. Therefore, this study was excluded from the sensitivity analysis. The pooled ORs were 7.15 (95\%CI: 1.34, 38.01), suggesting that NLR had a greater effect on CVD risk in study with shorter follow-up time than study with longer follow-up time.

Egger's test (Coefficient $=6.96 ; \mathrm{P}=0.017$ ) and funnel plot suggested publication bias; see Supplementary Figure 7B. A contour-enhanced funnel plot found that missing studies were in the nonsignificant area. Therefore, asymmetry in the funnel likely represents publication bias; see Supplementary Figure 7C.

\section{Discussion}

We conducted a systematic review and meta-analysis to assess the risk of NLR on CVD. Our study showed that high NLR was significantly associated with all CVD outcomes including CAD, ACS, stroke, and composite CVEs with pooled ORs ranging from 1.62 to 3.86. In addition, mean NLR was significantly higher in CVD patients than in controls, with USMDs ranging from 0.87 to 2.12 .

For the effect of high NLR on CVD outcomes, the strongest association was found in composite CVEs with pooled OR of 3.86, while the highest mean difference of NLR was found in ACS outcome with USMD of 2.12. However, ACS was the most common outcome defined in composite 
CVEs. Therefore, this may imply that the NLR effect was strongest on ACS outcome although consistent associations were seen across all individual CVD events. This may be attributed to the fact that NLR was associated with both acute and chronic forms of the atherosclerosis process.

There are various possible mechanisms that can explain the relationship between elevated NLR and risk of cardiovascular events. Neutrophils secrete inflammatory mediators that can lead to vascular wall degeneration [50]. Conversely, lymphocytes regulate the inflammatory response and have an antiatherosclerotic role in which regulatory T-cell, a subclass of lymphocyte, may have an inhibitory effect on atherosclerosis [51]. Previous studies also showed that a low lymphocyte count served as an early marker of physiologic stress and systemic collapse secondary to myocardial ischemia mediated by cortisol release [52, 53]. Increased cortisol levels result in a reduction in the relative level of lymphocytes [54].

Prior evidence has shown that high NLR is significantly associated with progression of atherosclerosis [55] and is also an independent predictor of thin-cap fibroatheroma [56]. Neutrophil infiltration into atherosclerotic plaques has also been found in atherectomy specimens of ACS patients and may contribute to its destabilization [57]. Activated neutrophils are known to release a variety of proteolytic enzymes [58]; neutrophil elastase in particular has been shown to mediate both degradation of basement membrane constituents and endothelial damage [59].

The C-reactive protein (CRP), one of the inflammatory biomarkers, has been investigated and found to be strongly associated with the risk and prognosis of CVDs [48, 60-62]. It also has positive correlation with neutrophil, monocyte, and NLR $[25,60]$. Previous studies illustrated that NLR could be a potential surrogate marker of systemic inflammation in its ability to predict hs-CRP [63] and CRP levels [64]. Therefore, NLR might not have only the direct effect on CVD risk but also have the indirect effect via CRP level. On the contrary, NLR might not be independently associated with CVD. The significant association between NLR and CVD may be confounded by CRP. However, to prove these hypotheses, further study that measured both NLR and CRP level is required.

Our study has some strengths. To the best of our knowledge, this is the first systematic review and meta-analysis to address the association between NLR and risk of CVD. The analysis was based on studies with relatively large sample sizes from diverse countries. All components of CVD including CAD, ACS, stroke, and TIA were included. In addition, the effect of high NLR and mean difference of NLR between CVD and non-CVD patients were estimated in our analysis.

However, some limitations could not be avoided. Most included studies were cross-sectional studies. Therefore, a causal link between NLR and risk of CVD could not be confirmed. Moreover, the eligible studies used various cutoff values for classifying high versus low NLRs. Although we recategorized these cut-off points based on aggregated data, there was still some overlap in cut-off points between low and high NLRs across studies. This discrepancy might cause moderate to high heterogeneity in some of our pooled estimates. Individual patient data meta-analysis would be more flexible for addressing this problem.

In addition, the Framingham Risk Score was shown to overestimate risk of coronary heart disease risk in a general population from several ethnicities [65]. However, a previous study demonstrated that NLR can independently predict CHD mortality and reclassify people in the intermediate risk category of the Framingham Risk Score to a higher category [66]. Currently, there are numerous models predicting incident CVD in the general population but many lack external validation [67]. Including NLR may help to improve the performance of CVD risk prediction model. Therefore, future research should explore on comparing existing CVD risk models with those including NLR.

\section{Conclusion}

The present systematic review and meta-analysis suggest that high NLR was associated with CAD, ACS, stroke, and composite cardiovascular events. Therefore, NLR should be considered when assessing the cardiovascular risk in the population.

\section{Data Availability}

The data used to support the findings of this study are included within the article.

\section{Conflicts of Interest}

The authors declare that there are no conflicts of interest.

\section{Acknowledgments}

The authors are grateful to all staff of the Section for Clinical Epidemiology and Biostatistics, Faculty of Medicine Ramathibodi Hospital, Mahidol University, Thailand.

\section{Supplementary Materials}

Supplementary Appendix: search terms and search strategy used for Scopus and Medline database. Supplementary Table 1: risk of bias assessment (case-control study). Supplementary Table 2: risk of bias assessment (cohort and cross-sectional studies). Supplementary Figures: Supplementary Figure 1: high versus low neutrophil lymphocyte ratio and coronary artery disease. Supplementary Figure 2: mean differences of neutrophil lymphocyte ratio and coronary artery disease. Supplementary Figure 3: high versus low neutrophil lymphocyte ratio and acute coronary syndrome. Supplementary Figure 4: mean differences of neutrophil lymphocyte ratio and acute coronary syndrome. Supplementary Figure 5: high versus low neutrophil lymphocyte ratio and stroke. Supplementary Figure 6: mean differences of neutrophil lymphocyte ratio and stroke. Supplementary Figure 7: high versus low neutrophil lymphocyte ratio and composite cardiovascular events (CVEs). (Supplementary Materials) 


\section{References}

[1] "All WHO fact sheets: Cardiovascular diseases (CVDs). Fact sheet number 317," 2017, http://www.who.int/mediacentre/ factsheets/fs317/en/.

[2] “Top 10 Causes of Death. GBD Compare," 2016, http://viz .healthmetricsandevaluation.org/gbd-compare/.

[3] E. J. Benjamin, M. J. Blaha, S. E. Chiuve et al., "Heart Disease and Stroke Statistics-2017 Update: A Report From the American Heart Association," in Circulation, The American Heart Association, 2017.

[4] M. Hoffman, A. Blum, R. Baruch, E. Kaplan, and M. Benjamin, "Leukocytes and coronary heart disease," Atherosclerosis, vol. 172, no. 1, pp. 1-6, 2004.

[5] L. Venkatraghavan, T. P. Tan, J. Mehta, A. Arekapudi, A. Govindarajulu, and E. Siu, "Neutrophil Lymphocyte Ratio as a predictor of systemic inflammation - A cross-sectional study in a pre-admission setting," F1000Research, vol. 4, 2015.

[6] X. Wang, G. Zhang, X. Jiang, H. Zhu, Z. Lu, and L. Xu, "Neutrophil to lymphocyte ratio in relation to risk of all-cause mortality and cardiovascular events among patients undergoing angiography or cardiac revascularization: a meta-analysis of observational studies," Atherosclerosis, vol. 234, no. 1, pp. 206213, 2014.

[7] L. Guasti, F. Dentali, L. Castiglioni et al., "Neutrophils and clinical outcomes in patients with acute coronary syndromes and/or cardiac revascularisation. A systematic review on more than 34,000 subjects," Thrombosis and Haemostasis, vol. 106, no. 4, pp. 591-599, 2011.

[8] F. Dentali, O. Nigro, A. Squizzato et al., "Impact of neutrophils to lymphocytes ratio on major clinical outcomes in patients with acute coronary syndromes: A systematic review and metaanalysis of the literature," International Journal of Cardiology, vol. 266, pp. 31-37, 2018.

[9] D. F. Stroup, J. A. Berlin, S. C. Morton et al., "Meta-analysis of observational studies in epidemiology: a proposal for reporting," Journal of the American Medical Association, vol. 283, no. 15, pp. 2008-2012, 2000.

[10] O. Sönmez, G. Ertaş, A. Bacaksiz et al., "Relation of neutrophil -to- lymphocyte ratio with the presence and complexity of coronary artery disease: an observational study," Anadolu Kardiyoloji Dergisi, vol. 13, no. 7, pp. 662-667, 2013.

[11] S. Naz, Z. Ali, and B. Akhtar, "Neutrophil lymphocyte ratio in coronary artery disease," Pakistan Journal of Medical and Health Sciences, vol. 8, no. 1, pp. 68-70, 2014.

[12] F. A. Mayyas, M. I. Al-Jarrah, K. S. Ibrahim, and K. H. Alzoubi, "Level and significance of plasma myeloperoxidase and the neutrophil to lymphocyte ratio in patients with coronary artery disease," Experimental and Therapeutic Medicine, vol. 8, no. 6, pp. 1951-1957, 2014.

[13] I. Sari, M. Sunbul, C. Mammadov et al., "Relation of neutrophilto-lymphocyte and platelet-to-lymphocyte ratio with coronary artery disease severity in patients undergoing coronary angiography," Kardiologia Polska, vol. 73, no. 12, pp. 1310-1316, 2015.

[14] F. Aygün and D. Efe, "Association of neutrophil/lymphocyte ratio with obstructive coronary artery disease and coronary artery calcium score detected by multislice computed tomography in type 2 diabetes mellitus patients," Patient Preference and Adherence, vol. 9, pp. 1023-1031, 2015.
[15] G. Açar, S. Fidan, Z. A. Uslu et al., "Relationship of neutrophillymphocyte ratio with the presence, severity, and extent of coronary atherosclerosis detected by coronary computed tomography angiography," Angiology, vol. 66, no. 2, pp. 174-179, 2015.

[16] M. Verdoia, A. Schaffer, L. Barbieri et al., "Impact of diabetes on neutrophil-to-lymphocyte ratio and its relationship to coronary artery disease," Diabetes \& Metabolism, vol. 41, no. 4, pp. 304311, 2015.

[17] F. Gungoren, F. Besli, S. Caliskan, U. Polat, C. Basar, and O. A. Serdar, "Statin Therapy May not Effect NLR and MPV Levels in Patients with Hypercholesterolemia," Angiology, vol. 67, no. 6, pp. 536-540, 2015.

[18] C. Yu, M. Chen, Z. Chen, and G. Lu, "Predictive and prognostic value of admission neutrophil-to-lymphocyte ratio in patients with CHD," Herz, vol. 41, no. 7, pp. 605-613, 2016.

[19] M. L. Perl, A. Finkelstein, M. Revivo et al., "Variance in biomarker usefulness as indicators for carotid and coronary atherosclerosis," Israel Medical Association Journal, vol. 18, pp. 80-84, 2016.

[20] M. Verdoia, L. Barbieri, G. Di Giovine, P. Marino, H. Suryapranata, and G. De Luca, "Neutrophil to lymphocyte ratio and the extent of coronary artery disease: results from a large cohort study," Angiology, vol. 67, no. 1, pp. 75-82, 2016.

[21] H. B. Uysal, B. Dağlı, C. Akgüllü et al., "Blood count parameters can predict the severity of coronary artery disease," Korean Journal of Internal Medicine, vol. 31, no. 6, pp. 1093-1100, 2016.

[22] M. Yılmaz, H. Korkmaz, M. N. Bilen, Ö. Uku, and E. Kurtoğlu, "Could neutrophil/lymphocyte ratio be an indicator of coronary artery disease, coronary artery ectasia and coronary slow flow?" Journal of International Medical Research, vol. 44, no. 6, pp. 1443-1453, 2016.

[23] S. Chittawar, D. Dutta, Z. Qureshi, V. Surana, S. Khandare, and T. N. Dubey, "Neutrophil-lymphocyte ratio is a novel reliable predictor of nephropathy, retinopathy, and coronary artery disease in Indians with type-2 diabetes," Indian Journal of Endocrinology and Metabolism, vol. 21, no. 6, pp. 864-870, 2017.

[24] T. Guo, L. Huang, C. Liu et al., "The clinical value of inflammatory biomarkers in coronary artery disease: PTX3 as a new inflammatory marker," Experimental Gerontology, vol. 97, pp. 64-67, 2017.

[25] K. Sharma, A. K. Patel, K. H. Shah, and A. Konat, "Is Neutrophilto-Lymphocyte Ratio a Predictor of Coronary Artery Disease in Western Indians?" International Journal of Inflammation, vol. 2017, Article ID 4136126, 8 pages, 2017.

[26] A. Korkmaz, A. Yıldız, P. Türker Duyuler et al., "Combination of change in hematological parameters with exercise stress test to predict coronary artery disease," Journal of Clinical Laboratory Analysis, vol. 32, no. 1, p. e22205, 2018.

[27] A. D. Zazula, D. Précoma-Neto, A. M. Gomes et al., "An assessment of neutrophils/lymphocytes ratio in patients suspected of acute coronary syndrome," Arquivos Brasileiros de Cardiologia, vol. 90, no. 1, pp. 30-35, 2008.

[28] B. G. Nordestgaard, A. S. Adourian, J. J. Freiberg, Y. Guo, P. Muntendam, and E. Falk, "Risk factors for near-term myocardial infarction in apparently healthy men and women," Clinical Chemistry, vol. 56, no. 4, pp. 559-567, 2010.

[29] G. Caimi, R. Lo Presti, B. Canino, E. Ferrera, and E. Hopps, "Behaviour of the neutrophil to lymphocyte ratio in young subjects with acute myocardial infarction," Clinical Hemorheology and Microcirculation, vol. 62, no. 3, pp. 239-247, 2016. 
[30] C.-R. Qiu, Q. Fu, J. Sui et al., "Analysis of Serum Endothelial Cell-Specific Molecule 1 (Endocan) Level in Type 2 Diabetes Mellitus with Acute ST-Segment Elevation Myocardial Infarction and its Correlation," Angiology, vol. 68, no. 1, pp. 74-78, 2017.

[31] A. Nalbant, H. Cinemre, T. Kaya, C. Varim, P. Varim, and A. Tamer, "Neutrophil to lymphocyte ratio might help prediction of acute myocardial infarction in patients with elevated serum creatinine," PAkistan Journal of Medical Sciences, vol. 32, no. 1, pp. 106-110, 2016.

[32] M. Ugur Goktas, P. Y. Akyol, Z. Karakaya, U. Payza, and F. E. Topal, "Evaluation of white blood cell and neutrophil/lymphocyte ratio in acute coronary syndrome patients admitted to emergency department," Journal of Biomedical Research, vol. 29, no. 10, pp. 2009-2014, 2018.

[33] W. Saliba, O. Barnett-Griness, M. Elias, and G. Rennert, "Neutrophil to lymphocyte ratio and risk of a first episode of stroke in patients with atrial fibrillation: a cohort study," Journal of Thrombosis and Haemostasis, vol. 13, no. 11, pp. 1971-1979, 2015.

[34] G. Ertaş, O. Sönmez, M. Turfan et al., "Neutrophil/lymphocyte ratio is associated with thromboembolic stroke in patients with non-valvular atrial fibrillation," Journal of the Neurological Sciences, vol. 324, no. 1-2, pp. 49-52, 2013.

[35] A. Celikbilek, S. Ismailogullari, and G. Zararsiz, "Neutrophil to lymphocyte ratio predicts poor prognosis in ischemic cerebrovascular disease," Journal of Clinical Laboratory Analysis, vol. 28, no. 1, pp. 27-31, 2014.

[36] E. Akil, M. A. Akil, S. Varol et al., "Echocardiographic epicardial fat thickness and neutrophil to lymphocyte ratio are novel inflammatory predictors of cerebral ischemic stroke," Journal of Stroke and Cerebrovascular Diseases, vol. 23, no. 9, pp. 23282334, 2014.

[37] D. Wang, F. Zhang, Y. Zhao et al., "Association of polymorphism in ICAM-1 (K469E) and cytology parameters in patients' initial blood test with acute ischemic stroke," Genetics and Molecular Research, vol. 14, no. 4, pp. 15520-15529, 2015.

[38] E. Köklü, İ. Ö. Yüksel, Ş. Arslan et al., "Is elevated neutrophilto-lymphocyte ratio a predictor of stroke in patients with intermediate carotid artery stenosis?" Journal of Stroke and Cerebrovascular Diseases, vol. 25, no. 3, pp. 578-584, 2016.

[39] B. Suh, D. W. Shin, H. Kwon et al., "Elevated neutrophil to lymphocyte ratio and ischemic stroke risk in generally healthy adults," PLoS ONE, vol. 12, no. 8, p. e0183706, 2017.

[40] H. Long, K. Qin, J. Chen et al., "Biomarkers of gastric cancer-related ischemic stroke and its underlying pathogenesis," Medicine, vol. 97, no. 17, p. e0493, 2018.

[41] I. Abete, M. A. Zulet, E. Goyenechea et al., "Association of lifestyle, inflammatory factors, and dietary patterns with the risk of suffering a stroke: A case-control study," Nutritional Neuroscience, vol. 21, no. 1, pp. 70-78, 2018.

[42] R. Farah and N. Samra, "Mean platelets volume and neutrophil to lymphocyte ratio as predictors of stroke," Journal of Clinical Laboratory Analysis, vol. 32, no. 1, 2018.

[43] J. C.-R. Tsai, S.-H. Sheu, H.-C. Chiu et al., "Association of peripheral total and differential leukocyte counts with metabolic syndrome and risk of ischemic cardiovascular diseases in patients with type 2 diabetes mellitus," Diabetes/Metabolism Research and Reviews, vol. 23, no. 2, pp. 111$118,2007$.

[44] B. Azab, V. Chainani, N. Shah, and J. T. McGinn, "Neutrophillymphocyte ratio as a predictor of major adverse cardiac events among diabetic population: a 4-year follow-up study," Angiology, vol. 64, no. 6, pp. 456-465, 2013.

[45] Y. Solak, M. I. Yilmaz, A. Sonmez et al., "Neutrophil to lymphocyte ratio independently predicts cardiovascular events in patients with chronic kidney disease," Clinical and Experimental Nephrology, vol. 17, no. 4, pp. 532-540, 2013.

[46] T. Abe, S. Kato, Y. Tsuruta et al., "Neutrophil/lymphocyte ratio as a predictor of cardiovascular events in incident dialysis patients: a Japanese prospective cohort study," Clinical and Experimental Nephrology, vol. 19, no. 4, pp. 718-724, 2015.

[47] E. Quiros-Roldan, E. Raffetti, F. Donato et al., "Neutrophil to Lymphocyte Ratio and Cardiovascular Disease Incidence in HIV-Infected Patients: A Population-Based Cohort Study," PLoS ONE, vol. 11, no. 5, p. e0154900, 2016.

[48] J. Danesh, J. G. Wheeler, G. M. Hirschfield et al., "C-reactive protein and other circulating markers of inflammation in the prediction of coronary heart disease," The New England Journal of Medicine, vol. 350, no. 14, pp. 1387-1397, 2004.

[49] C. K. Lo, D. Mertz, and M. Loeb, "Newcastle-Ottawa Scale: comparing reviewers' to authors' assessments," BMC Medical Research Methodology, vol. 14, no. 1, 2014.

[50] U. Ikeda, M. Ikeda, T. Oohara, S. Kano, and T. Yaginuma, "Mitogenic action of interleukin- $1 \alpha$ on vascular smooth muscle cells mediated by PDGF," Atherosclerosis, vol. 84, no. 2-3, pp. 183-188, 1990.

[51] E. Simpson and H. Cantor, "Regulation of the immune response by subclasses of T lymphocytes. II. The effect of adult thymectomy upon humoral and cellular responses in mice," European Journal of Immunology, vol. 5, no. 5, pp. 337-343, 1975.

[52] S. R. Ommen, R. J. Gibbons, D. O. Hodge, and S. P. Thomson, "Usefulness of the lymphocyte concentration as a prognostic marker in coronary artery disease," American Journal of Cardiology, vol. 79, no. 6, pp. 812-814, 1997.

[53] E. G. Zouridakis, X. Garcia-Moll, and J. C. Kaski, "Usefulness of the blood lymphocyte count in predicting recurrent instability and death in patients with unstable angina pectoris," American Journal of Cardiology, vol. 86, no. 4, pp. 449-451, 2000.

[54] S. P. Thomson, L. J. McMahon, and C. A. Nugent, "Endogenous cortisol: A regulator of the number of lymphocytes in peripheral blood," Clinical Immunology and Immunopathology, vol. 17, no. 4, pp. 506-514, 1980.

[55] N. Kalay, O. Dogdu, F. Koc et al., "Hematologic parameters and angiographic progression of coronary atherosclerosis," Angiology, vol. 63, no. 3, pp. 213-217, 2012.

[56] Y. H. Choi, Y. J. Hong, Y. Ahn, I. H. Park, and M. H. Jeong, "Relationship between neutrophil-to-lymphocyte ratio and plaque components in patients with coronary artery disease: Virtual histology intravascular ultrasound analysis," Journal of Korean Medical Science, vol. 29, no. 7, pp. 950-956, 2014.

[57] T. Naruko, M. Ueda, K. Haze et al., "Neutrophil infiltration of culprit lesions in acute coronary syndromes," Circulation, vol. 106, no. 23, pp. 2894-2900, 2002.

[58] B. D. Horne, J. L. Anderson, J. M. John et al., "Which white blood cell subtypes predict increased cardiovascular risk?" Journal of the American College of Cardiology, vol. 45, no. 10, pp. 1638-1643, 2005.

[59] J. L. Dinerman, J. L. Mehta, T. G. P. Saldeen et al., "Increased neutrophil elastase release in unstable angina pectoris and acute myocardial infarction," Journal of the American College of Cardiology, vol. 15, no. 7, pp. 1559-1563, 1990. 
[60] J. Danesh, R. Collins, P. Appleby, and R. Peto, "Association of fibrinogen, C-reactive protein, albumin, or leukocyte count with coronary heart disease: meta-analyses of prospective studies," Journal of the American Medical Association, vol. 279, no. 18, pp. 1477-1482, 1998.

[61] S. Kaptoge, E. Di Angelantonio, G. Lowe, and et al, "C-reactive protein concentration and risk of coronary heart disease, stroke, and mortality: an individual participant meta-analysis," The Lancet, vol. 375, no. 9709, pp. 132-140, 2010.

[62] Emerging Risk Factors Collaboration, S. Kaptoge, and E. Di Angelantonio, "C-reactive protein, fibrinogen, and cardiovascular disease prediction," The New England Journal of Medicine, vol. 367, no. 14, pp. 1310-1320, 2012.

[63] E. Ahbap, T. Sakaci, E. Kara et al., "Neutrophil-to-lymphocyte ratio and platelet-to-lymphocyte ratio in evaluation of inflammation in end-stage renal disease," Clinical Nephrology, vol. 85, no. 4, pp. 199-208, 2016.

[64] R. Malhotra, D. Marcelli, G. von Gersdorff et al., "Relationship of Neutrophil-to-Lymphocyte Ratio and Serum Albumin Levels with C-Reactive Protein in Hemodialysis Patients: Results from 2 International Cohort Studies," Nephron, vol. 130, no. 4, pp. 263-270, 2015.

[65] Asia Pacific Cohort Studies Collaboration, F. Barzi, A. Patel et al., "Cardiovascular risk prediction tools for populations in Asia," Journal of Epidemiology and Community Health, vol. 61, no. 2, pp. 115-121, 2007.

[66] N. Shah, V. Parikh, N. Patel et al., "Neutrophil lymphocyte ratio significantly improves the Framingham risk score in prediction of coronary heart disease mortality: Insights from the National Health and Nutrition Examination Survey-III," International Journal of Cardiology, vol. 171, no. 3, pp. 390-397, 2014.

[67] J. A. Damen, L. Hooft, E. Schuit et al., "Prediction models for cardiovascular disease risk in the general population: systematic review," BMJ, p. i2416. 


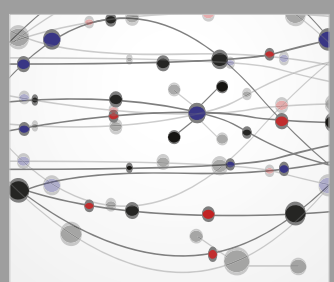

The Scientific World Journal
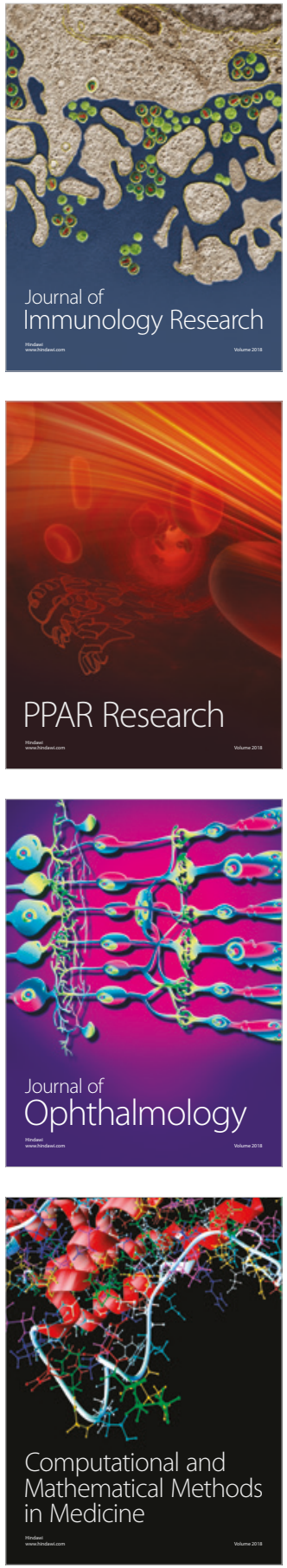

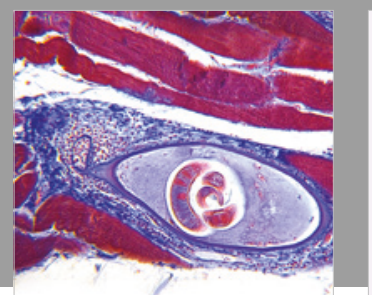

Gastroenterology Research and Practice

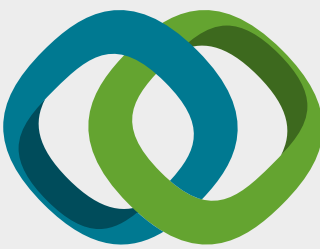

\section{Hindawi}

Submit your manuscripts at

www.hindawi.com
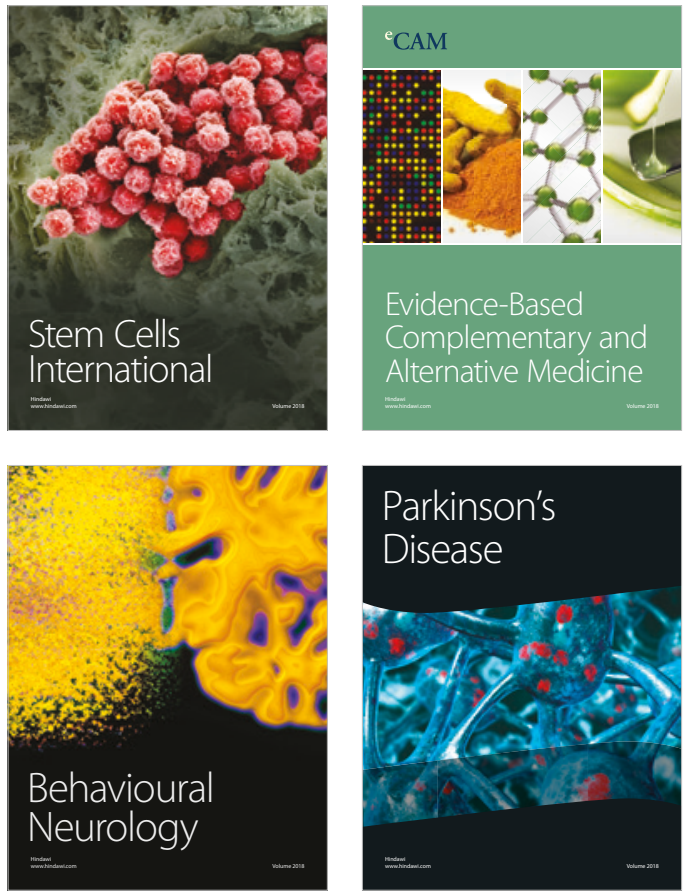

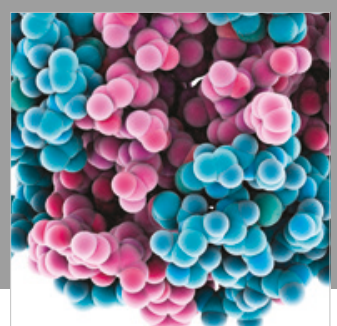

ournal of

Diabetes Research

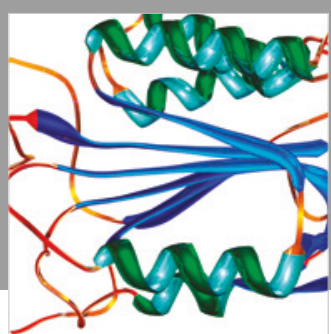

Disease Markers
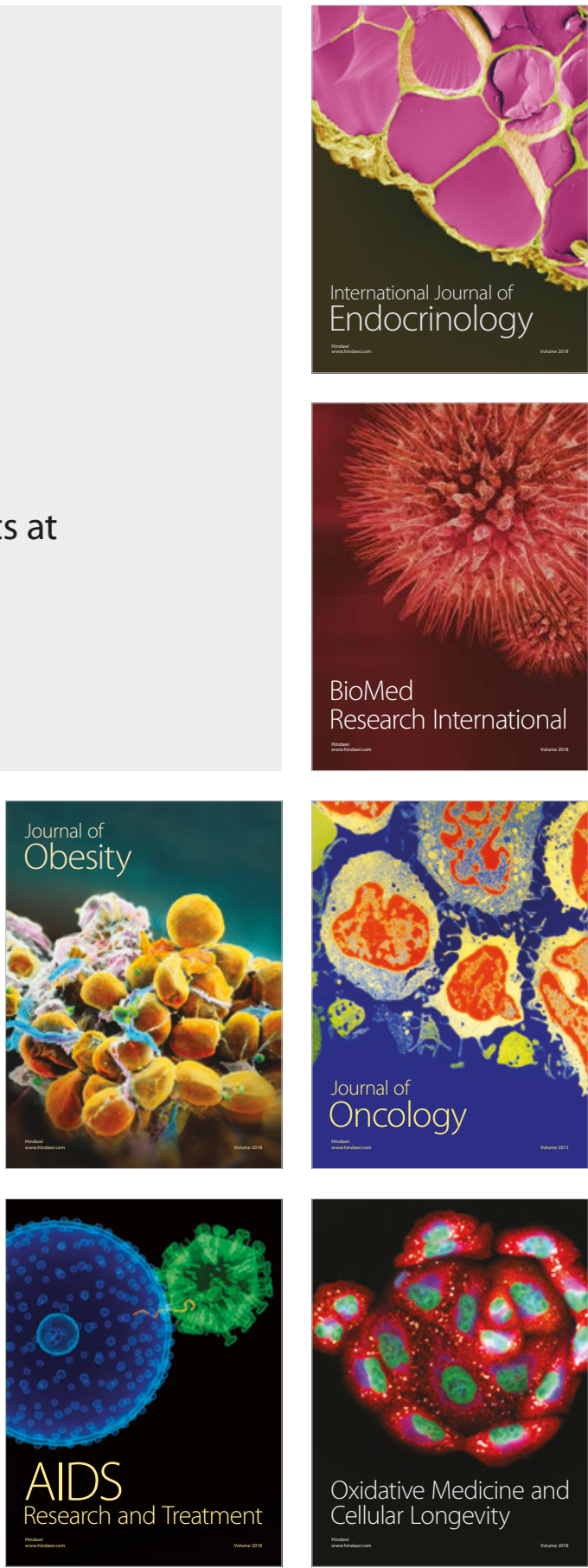